\title{
The CMS trigger in Run 2
}

\author{
Mia Tosi*t \\ CERN \\ E-mail: mia.tosi@cern.ch
}

During its second period of operation (Run 2) which started in 2015, the LHC will reach a peak instantaneous luminosity of approximately $2 \times 10^{34} \mathrm{~cm}^{-2} s^{-1}$ with an average pile-up of about 55 , far larger than the design value. Under these conditions, the online event selection is a very challenging task. In CMS, it is realised by a two-level trigger system: the Level-1 (L1) Trigger, implemented in custom-designed electronics, and the High Level Trigger (HLT), a streamlined version of the offline reconstruction software running on a computer farm.

In order to face this challenge, the L1 trigger has undergone a major upgrade compared to Run 1 , whereby all electronic boards of the system have been replaced, allowing more sophisticated algorithms to be run online. Its last stage, the global trigger, is now able to perform complex selections and to compute high-level quantities, like invariant masses. Likewise, the algorithms that run in the HLT went through big improvements; in particular, new approaches for the online track reconstruction lead to a drastic reduction of the computing time, and to much improved performance. This presentation will describe the performance of the upgraded trigger system in Run 2.

EPS-HEP 2017, European Physical Society conference on High Energy Physics 5-12 July 2017

Venice, Italy

\footnotetext{
* Speaker.

${ }^{\dagger}$ on behalf of the CMS collaboration
} 


\section{Introduction}

CMS [1] has a wide physics program for Run 2 (re-discovery of the Standard Model at $13 \mathrm{TeV}$, search of possible new physics, precision measurements of rare processes), therefore the main goal of the CMS trigger system is to keep the largest possible number of interesting events for analyses while keeping the event rate within the system limitation, namely $1 \mathrm{kHz}$.

The Run 2 data taking operations restarted at a centre-of-mass energy of $13 \mathrm{TeV}$ in 2015 with $25 \mathrm{~ns}$ proton bunch-spacing and an instantaneous luminosity which reached a peak value of $1.5 \times 10^{34} \mathrm{~cm}^{-2} \mathrm{~s}^{-1}$. It is expected to reach $2.0 \times 10^{34} \mathrm{~cm}^{-2} \mathrm{~s}^{-1}$ along with an average number of overlapping proton-proton interactions (pile-up, PU) of 60. In these conditions the CMS detector is crossed by thousands of particles in each bunch crossing. Such a high occupancy environment represents great challenges to the detector and its sub-systems.

During the two years long period of maintenance and upgrade activities, many improvements have been developed, in particular a flexible trigger system has been implemented capable of being adapted to the evolving CMS physics programme. In addition, during the extended year-end technical stop of the LHC 2016/2017, CMS has replaced its vertex detector. The new system is designed to withstand the harsh conditions of the LHC until the end of LHC Run 3. The detector has a four-hit coverage of up to $|\eta|<2.5$, with the first layer placed closer to the interaction point ( $\mathrm{r}=$ $29 \mathrm{~mm}$ ) and the new additional fourth layer further outside compared to the original pixel detector, allowing a more precise extrapolation of the tracks to the interaction region. This will allow for better vertexing and tracking capabilities, which turn into an improvement in the b-tagging performance.

In order to maintain and even improve the performance of the Run 1 trigger system [2], while keeping the rate (and timing) under control, a new trigger strategy has been defined, relying mainly on the pile-up dependence mitigation, a better matching to offline object reconstruction and taking advantage of the upgraded systems.

\section{The CMS trigger system}

The collision rate at the LHC is heavily dominated by large cross section QCD processes, which are not of prime interest for the physics program of the CMS experiment. The processes relevant for new physics usually occur at a rate smaller than $10 \mathrm{~Hz}$. Since it is not possible to record all the events, because of a limited bandwidth, it becomes mandatory to use a trigger system to select events according to physics-driven choices.

The CMS experiment features a two-level trigger architecture. The firmware based Level 1 Trigger (L1T) reduces the event rate from $40 \mathrm{MHz}$ to $100 \mathrm{kHz}$, and the software-based High Level Trigger (HLT) further reduces the rate to $1 \mathrm{kHz}$. L1T receives the information coming from the electromagnetic and hadronic calorimeters as well as from the muon chambers. A global decision is taken based on the presence of energy deposits compatible with physics objects such as photons, electrons, muons, jets or hadronically decaying tau leptons. The HLT is a streamlined version of the CMS offline reconstruction software running on a cluster of commercial rack-mounted computers which consists of about 26,000 processor cores.

\section{3. $\mathbf{L} 1$ performance in Run 2}

The key conceptual changes to the design of the L1T in Run 2 can be summarised as: 
- muon system - the redundancy of the three muon detectors (DT, RPC and CSC) is used already in the trigger primitives to make a high resolution muon trigger;

- calorimeter system - boundaries between the different detectros have been removed by streaming data from the same event into one FPGA;

- global trigger - expandable to many more possible conditions and compute more complex quantities, to give a richer menu [3].

The key technology and system updates are the replacement of the Run 1 VME-based electronics with $\mu$ TCA one; the use of latest generation of FPGAs, Xilinx Virtex 7; serial optical links in place of parallel copper ones (link speeds increased from $1.2 \mathrm{~Gb} / \mathrm{s}$ to $10 \mathrm{~Gb} / \mathrm{s}$ ); large optical patch panels with single fibres replaced with custom-made commercial solution (Molex Flexplane); online software rewritten to have more common code, modern libraries, more easily maintained [4].

The upgraded e/ $\gamma$ finding algorithm is based on dynamic clustering [5], allowing the dimensions of the energy clustered to form the e/ $\gamma$ candidate to adapt to the pattern of energy deposited in the electromagnetic calorimeter. This new approach provides a better energy containment for showering electrons and photon conversions, without being affected significantly by pile-up. Clusters produced using this algorithm have better energy resolution than those formed by the Run 1 algorithm, which used sliding window. The summed energy of the seed and clustered towers are used to compute the raw transverse energy $\left(E_{T}\right)$, and a calibration is applied which is dependent on the $\eta$ position of the seed tower and on the cluster shapes. In addition, the shape of the clusters may be used, along with the fraction of the total energy measured as electromagnetic, to discriminate between real e/ $\gamma$ candidates and background from hadronic jets, where the latter usually contain more trigger towers (TT). Isolation annuli (removing the footprint of the e/ $\gamma$ candidate) are constructed around each candidate cluster and are used to implement an isolation requirement, as a function of cluster $E_{T}$ and the PU estimated in each event. The performance of the upgraded L1 trigger system is checked with data. Figure 1 shows the measured trigger efficiency for the single e/ $\gamma$ triggers in Run 2, where a logical OR among different bits is used. In order to minimise the rate while keeping the lowest $E_{T}$ threshold, bits with lower $E_{T}$ thresholds have tighter $\eta$ acceptance and isolation working points. The improved energy calibration, $\mathrm{H} / \mathrm{E}$ veto and isolation re-optimisation for the 2017 data taking allowed to reduce the $E_{T}$ threshold by $2 \mathrm{GeV}$ with respect to the 2016 working point. The efficiency was measured using the tag-and-probe technique on a sample of $Z \rightarrow$ ee events and shows good efficiency at the plateau with a sharp turn-on, due to the excellent energy resolution [6].

Given the differences in background rate and magnetic field uniformity as $|\eta|$ increases, the muon track finding was segmented into three regions: Barrel $(|\eta|<0.83)$, Overlap $(0.83<|\eta|<$ 1.24) and Endcap $(|\eta|>1.24)$. A pattern-based track finding is used in the Endcap and Overlap regions, with a look-up-table based on an MVA for $p_{T}$-assignment. A simpler road search extrapolation track finder is used in the Barrel. The global muon trigger takes muon tracks from the regional finders, sorts them by muon $p_{T}$ and quality and removes any duplicate tracks found. Figure 2 shows the muon trigger efficiency for a single muon trigger with $p_{T}$ threshold of $25 \mathrm{GeV}$ as a function of the offline muon $p_{T}$ and $\eta$ [6]. The tag-and-probe method was used to measure the efficiency with a dataset of $\mathrm{Z} \rightarrow \mu \mu$ events. The improvement with respect to the 2016 setting comes from the inclusion of the RPC information in the Barrel and Endcap track finders as well as the retuning of the $p_{T}$-assignment. 


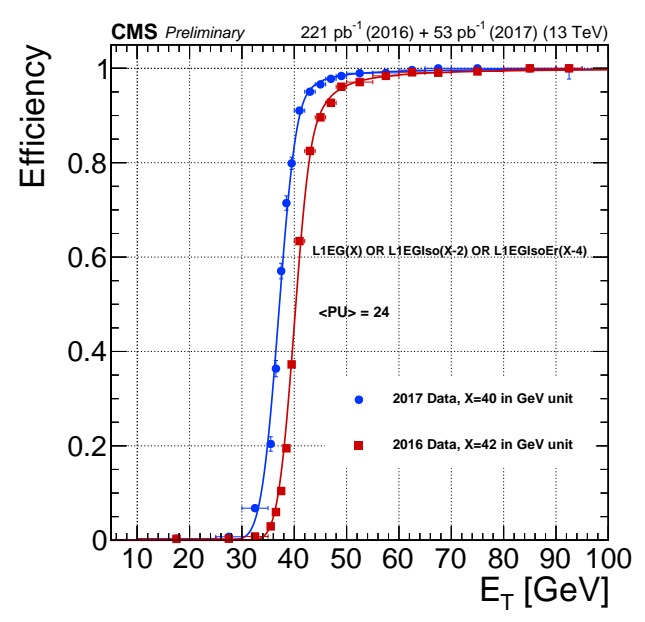

Figure 1: Trigger efficiency of the e/ $\gamma$ object as a function of the offline supercluster transverse energy $E_{T}$ with the 2017 algorithm (blue) and the 2016 algorithm (red) measured with tag-n-probe on data. The two curves are drawn for the thresholds $\mathrm{X}$ ensuring the same trigger rate [6].

The upgraded $\tau$-lepton finding algorithm is based on the same dynamic clustering as the e/ $\gamma$ finder described above, combining clusters (using ECAL and HCAL information) to build the hadronically decaying tau lepton candidate $\left(\tau_{h}\right)$. To improve the energy response and resolution, the L1 $\tau_{h}$ candidates are calibrated as function of the energy and $\eta$. To control the large rate of fake L1 $\tau_{h}$ candidates from QCD-induced jets, an upper cut on the L1 $\tau_{h}$ isolation energy is applied. The cut depends on the transverse energy and $\eta$ and a pile-up estimator, called nTT, which is defined as the number of trigger tower (TT) with non-zero energy in a central ring of $8 \mathrm{TT}$. The isolation energy cut values are built such that the efficiency to select a genuine $\tau$ is constant with $\eta$ and pile-up; the cut is relaxed at high $E_{T}$ to ensure $100 \%$ plateau efficiency.

The jet finding algorithm benefits from the increased spatial granularity of inputs compared to the Run 1 trigger. The algorithm is a 9x9 TT sliding window based sum, with dimensions matched to the $\mathrm{R}=0.4$ jet finding used offline by CMS. A jet is formed by searching for a seed energy deposit above a predefined threshold and summing the energy deposits around the seed. A veto mask is applied to remove duplicate jets and areas outside the size of typical jets are used to estimate and subtract the energy within the jet induced by the presence of PU. Jet energies are calibrated as a function of jet $E_{T}$ and $\eta$.

The Global Trigger has been re-implemented on modern FPGAs on an Advanced Mezzanine Card in a MicroTCA crate (uGT). The processing resources of the upgraded system with optical links running at $10 \mathrm{Gbit} / \mathrm{s}$ allows the implementation of a total of 512 algorithms at a time, instead of the 128 previously possible, allowing CMS to be more flexible in how it handles the trigger bandwidth. With this powerful processing units, more sophisticated quantities, such as the invariant mass of a pair of objects, can be computed. Different objects, e.g. muons with jets, can be matched with higher resolution and efficiency due to the upgraded calorimeter and muon trigger systems.

\section{HLT performance in Run 2}

The HLT menu in CMS has a modular structure. The menu is composed by more than 400 different HLT paths for the data taking in Run 2. Each path is a sequence of reconstruction and 
filtering modules, and it reproduces the offline selection for a given physics object (photons, electrons, muons, jets, missing energy, b-tagged jets, etc.), for combinations of them, or even for more sophisticated selections used in the physics analyses. The modules within a path are arranged in blocks of increased complexity, so that faster algorithms are run first and their products are filtered: if a filter fails, the rest of the path is skipped. There are other important features that differentiate the algorithms used at HLT to the ones used for the offline reconstructions, all meant to reduce the CPU time consumption at HLT: amongst them, the regionality (detector unpacking and reconstruction are restricted to regions of interest around the $\mathrm{L} 1$ or higher-level candidates), and the simplified track reconstruction.

Figure 3 shows the typical average timing per event of the HLT jobs run on the HLT farm as a function of the instantaneous luminosity as it was measured in 2016 data taking.

There are several improvements in the HLT algorithms and menu developed for Run 2. Overall, the most effective one is the usage of the Particle Flow methods and event reconstruction (PF) [8] in almost all HLT paths in CMS. The PF is a reconstruction technique widely used in CMS analyses. It makes use of the full detector information to describe the global collision event, by identifying particles individually and clustering them into more complex objects. PF at HLT improves the energy resolution of trigger objects and makes the online event reconstruction and selection much closer to the ones performed in the offline reconstruction and analyses. It provides in addition more efficient methods for PU mitigation. PU in fact affects the performance of many triggers: the additional tracks and calorimetric deposits tend to increase the rate of jet and missing transverse energy related triggers, as well as reduce the efficiency for the isolation algorithms.

Tracking certainly represents an essential ingredient for applying PF techniques to the whole event reconstruction. Tracking at HLT uses the same kind of iterative procedure as in the offline reconstruction. Such a procedure iteratively removes hits associated with tracks, reducing in this way the combinatorial in the subsequent steps. It starts with prompt, high $p_{T}$ tracks, then it looks for displaced ones, and only after for less frequent track topologies. In addition, searches for high mass resonances require tracks to have good momentum resolution for transverse momenta, $p_{T}$, of up to $1 \mathrm{TeV}$; it must also be possible to resolve very close tracks, such as those from 3-prong tau decay. Moreover, excellent impact parameter resolution is needed for a precise measurement of the primary vertex position and for b-jet identification. The CMS tracker was designed to satisfy these requirements and the track finding algorithms are designed to fully exploit its capabilities and deliver the desired performance.

A comparison of the tracking performance that can be achieved with the new pixel detector has been performed using simulated events. It suggests an increased tracking efficiency and a reduction of the fake rate by roughly a factor 4 over the whole $\eta$ range, as shown in Figure 4 . This has been achieved with the implementation of a new algorithm for the seeding step, called the Cellular Automaton (CA).

Precise measurements of the vertex positions and of the track impact parameters are crucial, to identify events with the presence of jets originating from the hadronisation of heavy flavour quarks. Such events are of primary interest in many physics analyses aiming at characterising the Higgs boson or searching for new physics beyond the Standard Model. The expected improvements in tracking and vertex reconstruction thanks to the pixel upgrade result in approximately $10 \%$ increase of the b-jet identification efficiency with the same fake rate level. 


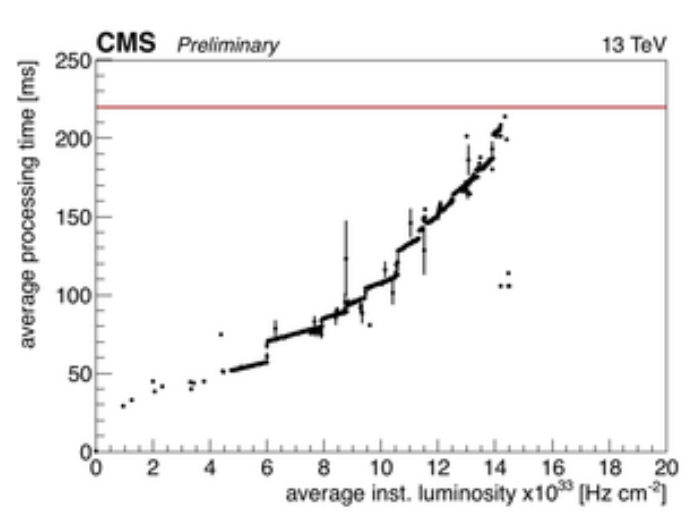

Figure 3: Average processing time of the HLT menu as function of the uncalibrated instantaneous luminosity, measured during data taking in fill 5393. The red line represents the HLT farm limit in 2016 [9].

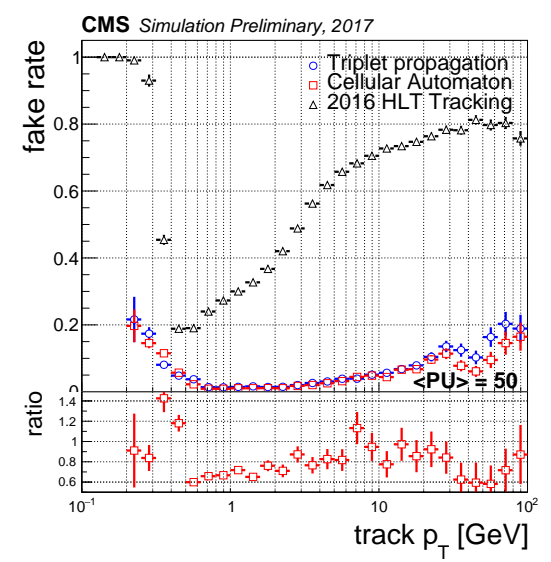

Figure 4: HLT tracking fake rate as function of the transverse momentum estimated with $t \bar{t}$ simulated events with average PU $=50$ for two different algorithms. Blue and red points refers to the new pixel detector, while the black ones to the old pixel detector [10].

\section{Conclusion}

With the increased luminosity and pile-up, the Run 2 at the LHC is a very challenging environment to carry out the CMS physics program. This was achieved by the upgrade of the L1 trigger system, which has been completed in 2015 and extensively commissioned and operated in 2016, and by the new developments in the physics objects reconstruction as well as the improvements in the algorithms implemented at HLT. These improvements took advantage of the new features of the new pixel detector, which was installed in the end of 2016.

\section{References}

[1] CMS Collaboration, JINST 3, S08004 (2008)

[2] CMS Collaboration, CMS The TRIDAS Project: Technical Design Report, Volume 1: The Trigger Systems, CERN-LHCC-2000-038, CMS-TDR-6 (2002), https : / cds . cern. ch/record/ 706847

[3] C. Wulz for the CMS Collaboration, Data analysis at CMS Level-1 Trigger level: Migrating complex selection algorithms from offline analysis and High-Level trigger to the trigger electronics, PoS EPS-HEP2017 (these proceedings)

[4] CMS Collaboration, CMS Technical Design Report for the Level-1 Trigger Upgrade, CERN-LHCC-2013-011, CMS-TDR-12 (2013), https: / / cds. cern. ch/record/1556311

[5] A. Zabi for the CMS Collaboration, Triggering on electrons, jets and tau leptons with the CMS upgraded calorimeter trigger for the LHC RUN II, JINST 11, C02008 (2016)

[6] CMS Collaboration, Level-1 E/Gamma and Muon performance on 2017 data, CMS-DP-2017-024, http: //cds. cern.ch/record/2273270

[7] CMS Collaboration, Level 1 Tau trigger performance in 2016 data and VBF seeds at Level 1 trigger, CMS-DP-2017-022, https: / / cds . cern. ch/record/2273268

[8] CMS Collaboration, Particle-flow reconstruction and global event description with the CMS detector, JINST 12, P10003 (2017)

[9] CMS Collaboration, HLT timing plots for 2016 data, https://twiki.cern.ch/twiki/bin/view/CMSPublic/HLTplotsCHEP2016

[10] CMS Collaboration, Plots on Pixel Phase I Upgrade, https://twiki.cern.ch/twiki/bin/view/CMSPublic/HLTplotsPixelupgrade 